\title{
Use of pharmacy dispensing data to measure adherence and identify nonadherence with oral hypoglycemic agents
}

\author{
Fong Sodihardjo-Yuen ${ }^{1} \cdot$ Liset van Dijk ${ }^{2} \cdot$ Michel Wensing ${ }^{3}$. \\ Peter A. G. M. De Smet ${ }^{1,3,4}$ - Martina Teichert ${ }^{1,3,5}$
}

Received: 23 August 2016 / Accepted: 13 October 2016/Published online: 28 October 2016

(C) The Author(s) 2016. This article is published with open access at Springerlink.com

\begin{abstract}
Purpose A framework for calculation of adherence for oral hypoglycemic agents (OHAs) based on data from healthinsurance claims is available. Pharmacy dispensing data aid identification of nonadherent patients in pharmacy practices. However, use of these data for calculation of OHA adherence requires additional methodological categories. We examined the impact of different methodological choices on estimation of OHA adherence using pharmacy dispensing data.

Methods Four methodological categories were added to the framework available to be used for adherence calculation with pharmacy dispensing data. Three adherence measures were defined to supply pharmacists with significant information on OHA use of their patients: (i) percentage of days covered by use periods of dispensed medication (PDC), (ii) mean rate of adherent patients with a PDC $\geq 80 \%$ (MRAP80), and (iii)
\end{abstract}

Electronic supplementary material The online version of this article (doi:10.1007/s00228-016-2149-3) contains supplementary material, which is available to authorized users.

Fong Sodihardjo-Yuen

f.sodihardjo-yuen@knmp.nl

1 Royal Dutch Pharmacists Association, 2514 JL The Hague, the Netherlands

2 Netherlands Institute for Health Services Research, Utrecht, the Netherlands

3 Radboud Institute for Health Sciences, Department of IQ Healthcare, Radboud University Medical Centre, Nijmegen, the Netherlands

4 Radboud Institute for Health Sciences, Department of Clinical Pharmacy, Radboud University Medical Centre, Nijmegen, the Netherlands

5 Department of Clinical Pharmacy \& Toxicology, Leiden University Medical Center, Leiden, the Netherlands mean number of nonadherent patients (MNNP80) per pharmacy with a PDC $<80 \%$. A basic scenario was developed from 16 methodological categories. Consequences of choices for different parameters within these categories on the scores of the three adherence measures were calculated from dispensing data between July 2013 and July 2014.

Results Data were available for 604,500 OHA users in 1737 community pharmacies in the Netherlands. For the basic scenario, mean PDC for OHA was $88.3 \%$. MRAP80 was $80.3 \%$, which corresponded to an average of 69 nonadherent patients per pharmacy. Different choices for parameter values resulted in score variations for PDC of 85.0-91.8\%, for MRAP80 of 75.3-86.1\%, and between 49 and 92 MNNP80 per pharmacy. Conclusion Sixteen methodological categories specified calculation of OHA adherence based on pharmacy dispensing data. Adherence scores expressed as percentages were relatively robust to variation in parameter values, but differed substantially for the absolute numbers of nonadherent patients per pharmacy.

Keywords Adherence calculation · Nonadherence · Pharmacy dispensing data $\cdot$ Oral hypoglycemic agents

\section{Introduction}

Nonadherence to prescribed medication has been identified to be a major source of suboptimal management of several chronic diseases [1-5]. A recent Cochrane review reported that prescription-refill records collected routinely are a more objective method to measure adherence than self-reporting by patients, and less expensive than Medication Event Monitoring Systems [6]. Based on prescription-refill records, adherence can be measured as average medication availability by the percentage of days covered by drug use periods of 
dispensed medication (PDC) or the mean rate of adherent patients for a certain threshold, chosen mostly at PDC $\geq 80 \%$ (MRAP80). For calculation of these measures, several methodological choices must be made. Dependent on these choices, a large variation in corresponding adherence scores has been reported [7-13].

Transparency in calculation of adherence scores is needed to assess the validity of results and to enable comparisons between different studies [14-18]. Wilke et al. defined 12 methodological categories and analyzed the influence of variation in parameter values within these categories on the prevalence of nonadherence for oral hypoglycemic agents (OHAs) with claims data from a health-insurance fund in Germany [7]. These categories addressed choices in patient inclusion (e.g., based on the diagnosis, a certain number of prescriptions, and new or prevalent users), duration of the observation period, and period of drug use. They dealt with periods of medication cessation and switching within drug classes, stockpiling, overuse and the threshold of minimum PDC to define nonadherence [7]. Wilke et al. showed that some parameters had a stronger effect on adherence scores than others. In particular, the availability of information on the prescribed daily doses (PDDs) used to calculate periods of drug use changed PDC by $25 \%$ [7]. Information on the PDD and the number of tablets dispensed can be used to calculate periods of drug use more accurately than counting the number of dispensations. Usually, information on PDDs is available from clinical prescribing or dispensation records, but is not always present in databases for healthinsurance claims. Another advantage of pharmacy dispensing data is that they enable pharmacists to identify patients for additional pharmaceutical care early as those who do not refill medication for their chronic disease in time. Community pharmacists have been shown to improve nonadherence to therapies for chronic disease using their dispensing data [19-23]. However, pharmacy dispensing data have a limitation in that adherence scores for patients are usually calculated from dispensations within individual pharmacies only and do not allow following up of patients for dispensations across different pharmacies (which is possible from health-insurance claims). Consequently, additional methodological categories are needed to identify patients with dispensations in several pharmacies ("drop-in" patients) and those who cease to visit the pharmacy because of a change of address, hospital admission, or death ("without any dispensations in a certain time period"). For OHA adherence, an additional methodological category for dealing with insulin besides OHA use is also helpful. According to guidelines, initiation of insulin usually involves cessation of all but one OHA used previously. Thus, a reduction in OHA use in combination with insulin initiation could be assessed incorrectly as "patient nonadherence." For implementation of additional pharmaceutical care to nonadherent patients in pharmacies, information on the absolute number of patients identified as being nonadherent per pharmacy is also important.
These theoretical considerations gave rise to develop additional methodological categories to calculate adherence scores for OHAs using pharmacy dispensing data.

The main objective of the present study was to calculate OHA adherence based on a methodological framework for three measures: (i) PDC, (ii) mean percentage of patients with a PDC $\geq 80$ (MPAP80), and (iii) mean number of nonadherent patients at a PDC $<80 \%$ (MNNP80) per pharmacy. In addition, the consequences of alternative methodological choices on the scores of these adherence measures were calculated.

\section{Methods}

\section{Ethical approval of the study protocol}

Data from pharmacists and patients were coded and anonymized before analyses. Use of observational data in descriptive retrospective studies in the Netherlands is not considered as an interventional trial according to Directive 2001/20/EC and Dutch legislation [24]. Therefore, the study protocol did not need to be submitted to a medical ethic committee for approval.

\section{Data sources}

In the Netherlands, OHAs are available by prescription only. All dispensations are registered in the computer system of the pharmacy per patient for health-insurance claims as well as for automated surveillance of medication use. Dispensing data from more than $90 \%$ of the 1981 community pharmacies in the Netherlands are delivered every month to the Foundation of Pharmaceutical Statistics (SFK) on a routine basis. These data provide detailed information on the drugs dispensed, including the codes from the Anatomic Therapeutic Chemical (ATC) system of the World Health Organization [25], the prescribed daily dosage (PDD), and the amount dispensed. From this information, periods of drug use can be calculated by dividing the amount dispensed by the PDD. Data on patient information in the SFK consist of an anonymous number from the computer system of the local pharmacy and information on the patient's sex and year of birth.

\section{Data collection}

Data were collected from the SFK for community pharmacies that had provided complete dispensing data from 1 July 2012 to 1 July 2014 . This period covered the study period from 1 July 2013 to 1 July 2014. The data history for the year before the study period was needed to state first OHA dispensings as those dispensings within the study period without a dispensing in the year before. From the community pharmacies included, patients who used at least one OHA (ATC-code A10B) within 
the study period were eligible to be included in adherence calculations. Medication could be tracked for a specific patient over time by a unique anonymous patient code within an individual pharmacy. Pharmacies with shared populations and using the same anonymous codes for their patients could label themselves as "pharmacy clusters" at SFK. In these specific cases, dispensations for these patients could be followed up across pharmacies within a cluster.

\section{Outcome measures}

Two adherence measures were calculated. The first was the PDC and was the average number of days covered by drug use periods of dispensed medication in relation to all days during the study period (calculated from the first day of drug use until the end of this period as a medication possession rate) $[7,26]$. The second measure was MRAP80 as the rate of OHA user with a PDC $\geq 80 \%$ within all OHA users in the study period. An additional outcome measure for the potential effort for the pharmacists to provide additional care for their nonadherent patients was defined. This is stated as the mean absolute number of nonadherent patients at a PDC $<80 \%$ (MNNP80) per pharmacy within the pharmacies included.

\section{Methodological categories to measure adherence using pharmacy dispensing data}

Four methodological categories were added to the methodological framework with 12 categories for data on healthinsurance claims as defined by Wilke et al. [7] as categories 13-16 (Table 1). Three categories were added with regard to the use of dispensing data per pharmacy for: drop-in patients; "actual patients" (patients with recent dispensations to ensure that patients continue to visit a particular pharmacy and did not, for example, move away or die); the possibility to followup patients for their dispensations from several community pharmacies within pharmacy clusters. A fourth category was added whether to include OHA users who also used insulin.

A basic scenario was formulated from all 16 categories. Each methodological category offered a choice of up to three parameter values. The choices in this scenario made were aimed to select patients nonadherent to OHA for additional pharmaceutical care and to prevent false-positive labeling for nonadherence (Table 1). The resulting basic case included all OHAs with at least one OHA dispensation during the study period (categories 1 and 3), as well as new users and those who stopped OHA use during the study period (categories 2 and 6), regardless of concomitant use of insulin (category 15) within the study year (category 4). For subjects who used several OHA classes concomitantly (category 10), PDC was calculated in three steps: (i) per patient as the medication possession rate for each OHA class, (ii) the arithmetic mean per patient of the different medication possession rates, and (iii) average of the arithmetic patient mean (category 7). Different OHA classes are used concomitantly in treatment of diabetes mellitus, so concomitant use of OHAs was considered in the adherence calculation instead of switching between drug classes (category 7). To prevent false-positive selection for nonadherence, only those patients that appeared to visit a particular pharmacy regularly (categories 11,13 , and 14) were included. Periods of drug use were calculated as PDC by dividing the number of dispensed OHA tablets by PDD (category 5). These periods were adjusted for early refills (category 8 ). To reflect nonadherence rather than overuse, adherence PDC scores were truncated at $100 \%$ (category 9). For MRAP, a $80 \%$ threshold was chosen, which corresponded to other OHA studies [25] (category 12). To assess the impact of these choices in the basic scenario on the three adherence measures, parameter values could be varied for nine methodological categories from our data (categories 2, 3, 7, 8, and 11-15).

\section{Analyses}

A mean value was calculated for each adherence measure. The range of PDC scores for individual patients was shown by 5 th and 95th percentiles. To examine further the validity of our assumptions, we undertook three subanalyses.

The first subanalysis determined if OHA cessation might have been due to insulin use (category 2b). For subjects with OHA use in the first 6 months of the study period but no OHA use in the final 6 months, it was investigated whether they used insulin after OHA cessation.

The second subanalysis explored whether users of one OHA class might have switched to another drug class (e.g., due to side effects) instead of using several OHA classes concomitantly (category 7). For OHA users in the first 6 months of the study period who started using another OHA class, whether they had ceased to use the earlier used OHA class was tested.

For the third subanalysis, the influence of clustered data that allowed follow-up of patients for their dispensations from different pharmacies (category 16) on adherence scores was assessed. This analysis could be done for pharmacies that had labeled themselves as pharmacy clusters in our database. Scores of adherence measures were compared with adherence scores calculated for individual pharmacies within these clusters.

\section{Results}

For adherence assessments during the study period, data were available from 1737 of 1981 community pharmacies $(88 \%)$ in the Netherlands. For the basic scenario, 604,500 OHA users were included (Table 2). Mean PDC with OHA use was 
Table 1 Methodological categories to calculate adherence and identify nonadherence from pharmacy dispensing data

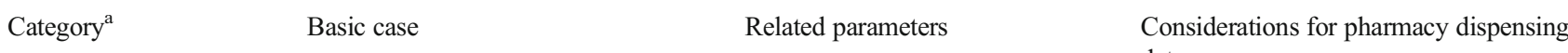
data

\begin{tabular}{|c|c|}
\hline $\begin{array}{l}\text { 1. Drug prescribing, } \\
\text { dispensing or diagnosis } \\
\text { as inclusion criterion }\end{array}$ & $\begin{array}{l}\text { Patient selection based on at least one } \\
\text { dispensing of } \mathrm{ATC}^{\mathrm{b}} \text { drug class A10B } \\
\text { within the study period }\end{array}$ \\
\hline
\end{tabular}

2. Patient type as inclusion All patients using at least one criterion

3. Minimum number of prescriptions as inclusion criterion
At least one dispensing during the study period dispensing of ATC class A10B within the study period, based on a dispensing in the study period or the 3 months prior a) Only prevalent users are included in adherence calculation, new users are excluded

b) Users that stopped with use of their medication are excluded

c) Only newly treated patients are included

At least two or more dispensings in the study period

\section{Observation period \\ 5. Period of drug use}

Annual analysis: July 2013-July 2014 Analysis for several months or years

Based on drug supply and prescribed daily doses
Based on a minimum number of dispensings
As pharmacy dispensing data lack information on diagnoses, diseases have to be estimated by dispensings. As oral hypoglycemic agents (OHA) are specifically used for type 2 diabetes mellitus, dispensing data can be used to select diabetes patients.

If information on diagnoses is available, the source can be used for more valid patient selection, e.g., specialist diagnosis

a) New users are defined as subjects with an OHA dispensing during the study period and no dispensing of any OHA drug during the prior 12 months

b) Stoppers are defined as subjects without a refill within the last 4 months of the study period. This excluded moved or ceased patients. The period of 4 months was chosen prescriptions generally are dispensed for 3 months

c) To choose when specific attention is paid to starting drug use

A higher number of dispensings could indicate that the therapy is chronic or that the patient is a permanent client of a community pharmacy. However, in nonadherence calculation, criteria for a higher number of dispensings induce bias by excluding patients who are not adherent after a small number of dispensings. To define permanent (no "drop-in") and actual pharmacy clients, other parameters can be used (see category 14 and 15 )

In the literature, observational periods of several months up to 10 years are used

Pharmacy dispensing data offer information on the prescribed daily dose (PDD). Thus periods of drug use can be calculated for distinct pharmaceutical formulations such as oral drugs by dividing the total number of dispensed drug units by the PDD.

Another, more basic way, for calculating nonadherence is to consider a patient as adherent when a specified number of prescriptions is refilled during the study period. Alternative methods of defining drug use periods, like what Wilke et al. applied, are based on the defined daily dose (DDD) or guideline recommendations. These approaches are however less accurate and useless when the PDD is available

With an interval-based nonadherence measure, the end of the interval is taken as a fixed date. This implicates that periods of drug discontinuation are included in the adherence measures. 
Table 1 (continued)

\begin{tabular}{|c|c|c|}
\hline Category $^{\mathrm{a}}$ & Basic case & Related parameters \\
\hline
\end{tabular}

data

\section{Adherence measure: assumption of concomitant use or switching between drugs/drug classes}

\section{Stockpiling \\ 9. Dealing with adherence Truncation to $100 \%$ $>100 \%$ on patient level \\ 10. Number of analyzed medication classes \\ No stockpiling considered \\ No truncation to $100 \%$ \\ Adherence measures for all medication classes for patients with mono- and multimedication, within OHA \\ Inclusion of only patients with monomedication or only analysis of one drug}

Concomitant use considered for different drug classes: estimation of the percentage of days covered with medication (PDC) separately calculated for the different OHA drug classes in use by one patient as arithmetic mean of the ATC group-specific PDCs
Switching considered between drug classes: estimation of the PDC as percentage of days covered by any OHA drug class
11. Absence periods (e.g., Exclusion of patients with absence hospital stay, holiday) periods
Inclusion of patients with absence periods

In dispensing-based calculation, discontinuation of therapy is not measured. To monitor treatment adherence of their patients, pharmacists also have to elucidate the reasons for treatment cessation, e.g., intentional, in agreement with the prescriber. Thus, a dispensing-based approach is not sufficient for pharmacists interventions

When assessing the PDC, it has to be decided on which ATC class level coverage of drug use is considered. This decision implies whether a patient is still adherent when switching to another drug, e.g., from metformin from one brand to another or also when switching from one OHA drug class to another (e.g., from metformin to glibenclamide). As in T2DM therapy, several OHA classes are used concomitantly, and thus metformin may rather have to be used concomitantly with glibenclamide

When accounting for stockpiling, periods of drug use are adjusted for early refills

With truncation, only nonadherence periods of underuse are considered with no periods of overuse. For OHA, overuse does not seem an issue

If adherence has to be calculated for a specific drug (class), adherence measures could be calculated for instance for metformin only within patients using metformin only or also additional OHA drugs. For pharmacists' interventions, the whole diabetes medication has to be taken into account. Thus, focus on one drug is not useful

When measuring adherence from the dispensings from one pharmacy only, patients might be falsely classified as nonadherent because of dispensings from other pharmacies, at hospital or during vacation. Periods of drug supplies from elsewhere are clearly visible in dispensing maps for patients using several chronic medications as simultaneous gaps in drug use. Patients with such gaps in their medication profiles can be excluded from adherence calculations to warrant the measures based on valid information

12. Threshold to calculate the mean rate of adherence patients

Threshold for nonadherence at an PDC $<80 \%$

Exclusion of "drop-in" patients exclusion criterion
Inclusion of "drop-in" patients
Threshold for nonadherence at PDC $<60$ or $<90 \%$ 
Table 1 (continued)

\begin{tabular}{|c|c|c|c|}
\hline Category $^{\mathrm{a}}$ & Basic case & Related parameters & $\begin{array}{l}\text { Considerations for pharmacy dispensing } \\
\text { data }\end{array}$ \\
\hline
\end{tabular}

consecutive days of any ATC drug class within 2 years.

Although the fidelity of Dutch patients to one community pharmacy is high, in urban or holiday regions, the number of patients who only once visit a pharmacy due to circumstances may be considerable. These would be falsely classified as nonadherent 14. "Actual patients" as Exclusion of non-actual patients $\quad$ Inclusion of non-actual patients
exclusion criterion
Inclusion of subjects using insulin concomitantly to OHA
16. Data source per
pharmacy or pharmacy

cluster
Individual community pharmacy
Exclusion of subjects using insulin concomitantly to OHA
Clustered data from several community pharmacies
Non-actual patients are subjects without a dispensing of any ATC drug class within the four last months at the end of the study period. As an example, patients who moved or died might falsely appear as nonadherent. These patients can be excluded as those without any dispensing in a pharmacy

T2DM users who cannot sufficiently be treated with three different OHA drug classes should receive insulin. They then may stop with all or some OHA drug classes. In this case, OHA discontinuation would be wrongly assumed as nonadherent. To avoid misclassification, insulin users could be excluded from nonadherence calculation. Use of insulin was defined by at least one dispensing of drug class A10A during the study period

Valid assessment of patients' adherence implies that all dispensings to a patient are taken into account. Using health-care claims databases, patient dispensings from several pharmacies are taken into account. However, when calculating patient's adherence by data from one pharmacy only, dispensings from other pharmacies might be missed

\footnotetext{
${ }^{a}$ Methodological categories 1-12 were derived from Wilke et al. [7] and expanded by 4 categories relevant for OHA nonadherence measurement by pharmacy dispensing data

${ }^{\mathrm{b}}$ Anatomic Therapeutic Chemical system of the World Health Organization

${ }^{\mathrm{c}}$ Possible parameter values for variation not used here for the calculation of adherence measures are printed in italic
}

$88.3 \%$ (5th percentile:95th percentile $=44.4 \%: 100 \%)$ and on average $80.3 \%$ of OHA users with a PDC $\geq 80 \%$ (MRAP80). These data corresponded to a mean number of 69 nonadherent patients (MNNP80) per pharmacy. Variation of parameter values within the 16 methodological categories resulted in score changes for all three adherence measures (Table 2). Mean adherence scores ranged between 85.0 and 91.8\% for the PDC, between 75.3 and 86.1\% for MRAP80, and between 49 and 92 for the MNNP80 per pharmacy (Table 2). Adherence rates increased (with a corresponding decrease in the absolute number of nonadherent patients per pharmacy) for parameter choices that excluded new users (category 2a), excluded gaps for ceased use of OHA (category 2b), or excluded OHA users with less than two dispensations (category 3). Adherence scores also increased if gaps in the use of one OHA class were filled with periods of drug use from another drug class and, hence, switching between OHA classes was considered instead of concomitant use (category 7). Finally, adherence scores improved if users of insulin besides OHA use were excluded (category 15).

Adherence rates decreased (with a corresponding increase in the absolute number of nonadherent patients per pharmacy) for choices of parameter values that did not consider stockpiling (category 4), included patients with periods of absence (category 5) or included drop-in patients (category 13) or included patients who did receive any drugs recently (category 14). 
Table 2 Influence of variation in parameter values on adherence measures for oral hypoglycemic agents

\begin{tabular}{|c|c|c|c|c|c|c|c|}
\hline \multirow[t]{2}{*}{ Category $^{\mathrm{a}}$} & \multirow[t]{2}{*}{ Parameter choice } & \multicolumn{2}{|l|}{ PDC } & \multicolumn{2}{|l|}{ MRAP80 } & \multirow{2}{*}{$\begin{array}{l}\text { MNNP80 per } \\
\text { pharmacy }\end{array}$} & \multirow{2}{*}{$\begin{array}{l}\text { Total number } \\
\text { of included } \\
\text { patients }\end{array}$} \\
\hline & & $\begin{array}{l}\text { Mean PDC }(5 \text { th; } \\
\text { 95th percentile) } \\
88.3(44.4 ; 100.0)\end{array}$ & $\begin{array}{l}\text { Difference } \\
\text { to basic case } \\
\text { NA }\end{array}$ & $\begin{array}{l}\text { Mean MRAP } \\
80.3\end{array}$ & $\begin{array}{l}\text { Difference } \\
\text { to basic case } \\
\text { NA }\end{array}$ & & \\
\hline \multicolumn{8}{|c|}{ Effects of parameter values variation compared to the basic case choices, mutually exclusive } \\
\hline $2 \mathrm{a}$ & $\begin{array}{l}\text { Only prevalent users, new } \\
\text { users of oral hypoglycemic } \\
\text { agents (OHA) are excluded }\end{array}$ & $89.1(50.9 ; 100.0)$ & +0.8 & 81.5 & +1.2 & 55 & 513,290 \\
\hline $2 b$ & Users ceasing OHA use excluded & $91.1(58.6 ; 100.0)$ & +2.8 & 84.7 & +4.4 & 50 & 567,704 \\
\hline 3 & $\begin{array}{l}\text { Users with a minimum number } \\
\text { of OHA dispensings }<2 \text { excluded }\end{array}$ & $90.3(55.9 ; 100.0)$ & +2.0 & 82.8 & +2.5 & 57 & 576,293 \\
\hline 7 & $\begin{array}{l}\text { Switching considered between } \\
\text { OHA drug classes }\end{array}$ & $91.8(49.6 ; 100.0)$ & +3.5 & 86.1 & +5.8 & 46 & 604,500 \\
\hline 8 & Stockpiling not considered & $85.0(41.9 ; 100.0)$ & -3.3 & 75.6 & -4.7 & 85 & 604,438 \\
\hline 11 & Subjects with absence periods included & $87.9(42.7 ; 100.0)$ & -0.4 & 79.5 & -0.8 & 72 & 611,702 \\
\hline 13 & Drop-in patients included & $88.1(43.0 ; 100.0)$ & -0.2 & 80.1 & -0.2 & 70 & 610,444 \\
\hline 14 & Patients without actual drug use included & $85.0(28.5 ; 100.0)$ & -3.3 & 75.3 & -5.0 & 92 & 649,004 \\
\hline 15 & Insulin users excluded & $88.9(47.4 ; 100.0)$ & +0.6 & 81.6 & +1.3 & 49 & 465,454 \\
\hline
\end{tabular}

Based on data from 1737 Dutch community pharmacies

${ }^{a}$ Numbering corresponding to the methodological categories introduced in Table 1

$P D C$ percentage of days covered by medication, MRAP80 mean rate of adherent patients with a PDC $\geq 80 \%$, MNNP80 per pharmacy mean number nonadherent patient at a PDC $<80 \%$ per pharmacy, $N A$ not applicable

Variation of the MRAP80 threshold for a stricter boundary of a mean medication possession rate of $\geq 90 \%$ (MRAP90) or a more liberal threshold of $60 \%$ (MRAP60) to be regarded as adherent resulted in changes of MRAP scores to $69.2 \%$ (MRAP90) and $90.8 \%$ (MRAP60) compared with the basic scenario of $80.3 \%$ (MRAP80) (category 12). This variation corresponded to a mean absolute number of 107 nonadherent patients (MNNP90) and 31 nonadherent patients (MNNP60) compared with 69 nonadherent patients per pharmacy (MNNP80).

The first subanalysis to ascertain if OHA cessation might have been due to insulin use (category 2b) involved 36,796 subjects with OHA use in the first 6 months of the study period and no use of OHAs in the final 6 months. From these 36,796 subjects, 3505 subjects $(9.5 \%)$ used insulin after OHA cessation. The other 33,291 subjects $(90.5 \%)$ did not receive insulin dispensations after OHA cessation. The second subanalysis (validity of the assumption for concomitant use of OHA classes instead of switching from one OHA class to another) involved 604,500 OHA users. From these 604,500 OHA users, 1932 subjects $(0.3 \%)$ were considered to be "switchers" due to starting a new OHA class after cessation of the previously used OHA class. For the third subanalysis (effect on adherence measures for following up patients for their dispensations across different pharmacies), 75 clusters were registered in the SFK database with 258 community pharmacies (13\% of all pharmacies) in the Netherlands, see supplementary information. On average, a cluster comprised 3.4 pharmacies. Following patients across different pharmacies increased all adherence scores for clustered data compared with data from individual pharmacies: for the basic scenario, PDC improved by $1.7 \%$ for clustered data compared with dispensations within individual pharmacies, MRAP80 improved by $2.8 \%$, and the MNNP per pharmacy decreased by 19 .

\section{Discussion}

Overall adherence scores calculated by pharmacy dispensing data were relatively robust to variations in parameters within a methodological framework for the PDC and percentage of subjects with a PDC $\geq 80 \%$ (MRAP80). Compared with a basic scenario defined by 16 methodological categories, different parameter choices within these categories changed the PDC by a maximum of $3.3 \%$ and MRAP 80 by a maximum of $5.8 \%$. These ranges were much lower than the nonadherence ranges of 16-97\% reported by Wilke et al. [7]. This variation was mainly due to whether or not information availability on the PDD. As the PDD was available in dispensing data, this reason for variation in adherence calculation was overcome by our data. However, numbers of OHA users per pharmacy were high, so these percentages corresponded with a substantial change (between 49 and 92) in the mean absolute numbers of nonadherent patients per pharmacy. The direction of changes in adherence scores from variations in parameter values were as expected from theoretical considerations. For 
instance, for MRAP, a variation in the threshold to label patients as "adherent" from at a PDC of 60,80 , or $90 \%$ had a considerable influence on the scores of adherence measures. In the literature, a PDC of $80 \%$ is commonly assumed to be sufficient to warrant the expected clinical outcomes. For OHA use, a PDC $<80 \%$ has been shown to be associated with an increased prevalence of hospitalization [26]. However, other cut-off points may be relevant depending on the specific drug treatment.

Another source of changes in adherence scores was the possibility of following patients according to their dispensations across different pharmacies by clustered data instead of using data per individual pharmacy. Use of dispensing data within individual pharmacies is a general limitation of pharmacy data. At present pooled data of different pharmacies can be used only in specific settings by pharmacies using the same anonymous codes for individual patients. If patients are likely to receive their medication from different pharmacies, the use of pooled data from pharmacy clusters can provide the individual pharmacists with more complete information on their patients' drug use. As expected, use of clustered data improved the scores of adherence measures, though changes were moderate for the PDC and MRAP80, with improvement of $\approx 3 \%$ for the basic scenario. However, for the basic scenario, the absolute numbers of patients identified for nonadherence decreased by on average 19 patients per pharmacy. This finding implies that, per pharmacy, 1 in 4 of the patients identified from data of individual pharmacies only were labeled falsely as being nonadherent due to dispensations from other pharmacies. It is probable that such pharmacies who registered as clusters shared their patient populations most with other community pharmacies and thus suffered most from incomplete information of their patients' drug use. Hence, our findings from this subgroup might have overestimated the effects of clustered data for adherence calculation.

Another source of considerable variation shown by Wilke et al. addressed category 6 for calculations of drug use within dispensings versus calculations until the fixed end of an interval (e.g., 1 year) [7]. This criterion resulted in changes in adherence scores by more than $7 \%$. Adherence calculations between dispensations per definition exclude periods of drug discontinuation and so tend to overestimate adherence in clinical practice. As for pharmaceutical care patients who stop their treatment for a chronic disease also need to be identified, variation in a parameter value for adherence within dispensations was not considered as a relevant alternative in our analysis. Nevertheless, cessation of one or more OHA might be related to the start of insulin treatment, in which case the prescriber stopped OHA use and so an observed discontinuation might not reflect nonadherence. As a limitation of our study, we did not dispose of information on the reason for drug discontinuation. To assess the potential resulting bias, our subanalysis showed that only about $10 \%$ of all subjects with OHA cessation used insulin subsequently. And even this limited percentage cannot be fully explained by prescriberinitiated OHA cessation as guidelines advise continuation of at least one OHA in combination with insulin. Our basic assumption to consider concomitant use rather than switching between different OHA classes was confirmed by a subanalysis which showed that only $0.3 \%$ of OHA users in the first 6 months of the study period switched to another OHA class.

In conclusion, a framework with 16 methodological categories was useful to define OHA adherence based on pharmacy dispensing data. Adherence scores expressed as percentages were relatively robust to variations in parameter values but differed substantially in terms of the absolute numbers of nonadherent patients per pharmacy.

\section{Acknowledgments None.}

Author contributions FS and MT designed the study. FS and MT collected data and undertook analyses. FS, MT, MW, PdS, and LvD wrote the manuscript.

\section{Compliance with ethical standards}

Conflict of interest The authors declare that they have no conflicts of interest.

Open Access This article is distributed under the terms of the Creative Commons Attribution 4.0 International License (http:// creativecommons.org/licenses/by/4.0/), which permits unrestricted use, distribution, and reproduction in any medium, provided you give appropriate credit to the original author(s) and the source, provide a link to the Creative Commons license, and indicate if changes were made.

\section{References}

1. van Boven J, Chavannes N, van der Molen T, Rutten-van Mölken M, Postma M, Vegter S (2014 Jan) Clinical and economic impact of non-adherence in COPD: a systematic review. Respir Med 108: 103-113

2. Simoni-Wastila L, Ryder P, Qian J, Zuckerman I, Shaffer T, Zhao L (2009) Association of antipsychotic use with hospital events and mortality among medicare beneficiaries residing in long-term care facilities. Am J Geriatr Psychiatry 17:417-427

3. Paterson D, Swindells S, Mohr J et al (2000) Adherence to protease inhibitor therapy and outcomes in patients with HIV infection. Ann Intern Med 133:2103

4. Penning-van Beest F, Erkens J, Olson M, Herings R (2008) Loss of treatment benefit due to low compliance with bisphosphonate therapy. Osteoporos Int 19:511-517

5. Ho PM, Rumsfeld JS, Masoudi FA et al (2006) Effect of medication nonadherence on hospitalization and mortality among patients with diabetes mellitus. Arch Intern Med 166:1836-1841

6. Nieuwlaat R, Wilczynski N, Navarro T, et al. Interventions for enhancing medication adherence (Review). The Cochrane Collaboration 2014;http://onlinelibrary.wiley.com/doi/10.1002 /14651858.CD000011.pub4/epdf. 
7. Wilke T, Groth A, Mueller S et al (2013) How to use pharmacy claims data to measure patient nonadherence? The example of oral diabetics in therapy of type 2 diabetes mellitus. Eur J Health Econ 14:551-568

8. Karve S, Cleves MA, Helm M, Hudson TJ, West DS, Martin BC (2008) An empirical basis for standardizing adherence measures derived from administrative claims data among diabetic patients. Med Care 46:1125-1133

9. Hess LM, Raebel MA, Conner DA, Malone DC (2006) Measurement of adherence in pharmacy administrative databases: a proposal for standard definitions and preferred measures. Ann Pharmacother 40:1280-1288

10. Cramer J, Benedict A, Muszbek N, Keskinaslan A, Khan Z (2007) The significance of compliance and persistence in the treatment of diabetes, hypertension and dyslipidaemia: a review. Int J Clin Pract 62:76-87

11. Lesén E, Sandström TZ, Carlsten A, Jönsson AK, Mårdby AC, Sundell KA (2011) A comparison of two methods for estimating refill adherence to statins in Sweden: the RARE project. Pharmacoepidemiol Drug Saf 20:1073-1079

12. Karve S, Cleves MA, Helm M, Hudson TJ, West DS, Martin BC (2009) Prospective validation of eight different adherence measures for use with administrative claims data among patients with schizophrenia. Value Health 12:989-995

13. Hansen R, Kim M, Song L, Ty W, Wu J, Murray DM (2009) Comparison of methods to assess medication adherence and classify nonadherence. Ann Pharmacother 43:413-422

14. Mantel-Teeuwisse AK, KO H, Monique VW, Porsius A, de Boer A (2001) Comparison of different methods to estimate prevalence of drug use by using pharmacy records. J Clin Epidemiol 54:11811186

15. Raebel MA, Schmittdiel J, Karter AJ, Konieczny JL, Steiner JF (2013 august) Standardizing terminology and definitions of medication adherence and persistence in research employing electronic databases. Med Care 51(8 0 3):S11-S21

16. Steiner JF, Prochazka AV (1997) The assessment of refill compliance using pharmacy records: methods, validity, and applications. J Clin Epidemiol 50:105-116
17. Andrade SE, Kahler KH, Frech F, Chan KA (2006) Methods for evaluation of medication adherence and persistence using automated databases. Pharmacoepidemiol Drug Saf 15:565-574

18. Arnet I, Kooij M, Messerli M, Hersberger K, Heerdink E, Bouvy ML. Proposal of standardization to assess adherence with medication recores: methodology matters. Annals of Pharmacotherapy 2016; 1060028016634106.

19. Stuurman-Bieze A, Hiddink E, van Boven J, Vegter S (2014) Proactive pharmaceutical care interventions decrease patients' nonadherence to osteoporosis medication. Osteoporos Int 25: $1807-1812$

20. Stuurman-Bieze AG, Hiddink EG, , Vegter S. Proactive pharmaceutical care interventions improve patients' adherence to lipid-lowering medication. Ann Pharmacother 2013; 47:1448-1456.

21. Vegter S, Oosterhof P, van Boven JF, Stuurman-Bieze AG, Hiddink EG, Postma MJ (2014) Improving adherence to lipid-lowering therapy in a community pharmacy intervention program: a cost-effectiveness analysis. J Manag Care Spec Pharm 20:722-732

22. van Boven J, Hiddink E, Stuurman-Bieze A, Chuiling-Veninga C, Postma M, Vegter S (2013) The pharmacists' potential to provide targets for interventions to optimize pharmacotherapy in patients with asthma. Int J Clin Pharm 35:1075-1082

23. Tommelein E, Mehuys E, Van Hees T et al (2014) Effectiveness of pharmaceutical care for patients with chronic obstructive pulmonary disease (PHARMACOP): a randomized controlled trial. Br J Clin Pharmacol 77:756-766

24. Nederlandse overheid. Wet medisch wetenschappelijk onderzoek met mensen. http://wetten.overheid.nl/BWBR0009408/2013-0927/1, accessed on October 11th 2016.

25. Methodology WCCfDS. Guidelines for ATC classification and DDD assignment 2013. http://www.whoccno/filearchive/publications/1_2013 guidelinespdf Oslo 2012.

26. Lau DT, Nau DP (2004) Oral antihyperglycemic medication nonadherence and subsequent hospitalization among individuals with type 2 diabetes. Diabetes Care 27:2149-2153 